\title{
Should a Replanted Avulsed Tooth Undergo Orthodontic Treatment?
}

\section{Zahida Parveen ${ }^{1 *}$, Akshata Ajay Shenvi ${ }^{2}$, Anand Krishnan ${ }^{3}$, Lisa Elizabeth Jacob ${ }^{4}$ and Deivanayagi ${ }^{5}$}

${ }^{1}$ Lecturer, Department of Pediatric Dentistry, Penang International Dental College, Penang, Malaysia

${ }^{2}$ Pediatric Dental Specialist, Navdurga Pediatric Dental Care, Mumbai, India

${ }^{3}$ Lecturer, Department of Oral Medicine and Radiology, Penang International

Dental College, Penang, Malaysia

${ }^{4}$ Senior Lecturer, Department of Oral Medicine and Radiology, Pushpagiri College

of Dental Sciences, Kerala, India

${ }^{5}$ Reader, Department of Oral Medicine and Radiology, Ragas Dental College and

Hospital, Tamil Nadu, India

*Corresponding Author: Zahida Parveen, Lecturer, Department of Pediatric

Dentistry, Penang International Dental College, Malaysia.
Received: August 21, 2021

Published: September 23, 2021

(C) All rights are reserved by Zahida Parveen., et al.

\begin{abstract}
Permanent tooth avulsion is the most devastating of all dental injuries, accounting for 1 - 16 percent of all traumatic injuries, with maxillary incisors being the most often affected. This case report documents the treatment and progress of an avulsed permanent right maxillary central incisor and extruded maxillary right lateral incisor undergoing orthodontic treatment. The patient had reported within 1 and half hours following trauma with the tooth stored in dry condition and soil contamination. The prognosis depends on measures taken at the place of the accident or the time frame within which the tooth is reimplanted after avulsion. Appropriate emergency management and treatment strategy are critical for a good prognosis.
\end{abstract}

Keywords: Avulsion; Avulsed Tooth; Dental Trauma; Replanted Tooth; Orthodontic Treatment

\section{Introduction}

Avulsion accounts for $0.5-16 \%$ of traumatic injuries in the permanent dentition [1]. Permanent tooth avulsion can occur at any age but is most common in young permanent dentition due to roots being incompletely formed and resilient periodontium and bone [2]. Shortly after avulsion, the prognosis primarily depends on the measures taken at the time and place of the accident [3]. Attachment damage, pulp necrosis, and small localized cemental damage occur when a tooth is avulsed. The Periodontal ligament
(PDL), if left attached to the root surface does not dry out and the unfavorable consequences of tooth avulsion are usually minimal. However, following replantation, if excessive drying of the PDL occurs, these PDL cells will trigger a severe inflammatory response, with physiologic re-contouring of bone on the root surface.

\section{Case Presentation}

A 16-year-old girl undergoing orthodontic treatment presented with an avulsed maxillary right central incisor, extruded maxillary 
right lateral incisor, and lip laceration due to Road Traffic Accident (RTA) approximately one and half hours back. The patient found the soiled avulsed tooth at the site of the accident and brought it to the M. S. Ramaiah Dental College, Department of Pediatric Dentistry in milk. Extra-oral and intraoral examination revealed swelling and edematous lips, missing right maxillary central incisor, grade I mobility of 21,22 , and grade II mobility of 12 . No fractures of the alveolar bone were detected in the clinical and radiographic examination of the tooth socket. The avulsed tooth was inspected for any debris or fracture. The root was completely formed and the crown portion of the avulsed tooth was intact. The tooth was laved in saline and the root surface was scraped with a Number 11 blade to detach the periodontal ligament remnants and debris. An IOPA of 11 was taken to detect the presence of fractures and morphology of the canal. Extra-oral access opening was done and pulp extirpated. The tooth was placed in Doxycycline for 10 minutes. The canal was irrigated with saline. MTA (Mineral trioxide aggregate) was placed in the canal at the apex. The socket of 11 was inspected for any loose bony fragments that would interfere with healing after administration of Local Anesthesia. The coagulum from the socket was removed using curettes. The tooth was placed back into the socket with light digital pressure. Tooth 12 , which had extruded, was gently reimplanted back into the socket.

Since the patient was already undergoing orthodontic treatment, the same Beggs Bracket and Ligature wire was used in the figure of eight extending from 12, 11, 21, and 22 for the stabilization. Splinting was done on the palatal surface. The patient was recalled after a week for obturation of 11.

After one-month post-operative apical resorption was noticed in relation to 12, hence Root canal therapy was done for the same.

\section{Discussion}

Tooth avulsion, or complete displacement of the tooth from its socket, causes damage to the periodontal ligament, alveolar bone, cementum, gingiva, and dental pulp. Complications of the avulsed teeth include pulp necrosis, internal/external root resorption, ankylosis, and loss of the periodontal ligament. As stated in the International association of dental traumatology (IADT) guidelines, incidence of complications depends on several factors, namely, transportation/storage of the teeth, the time that elapses between avulsion and replantation of the tooth, and splinting technique. In

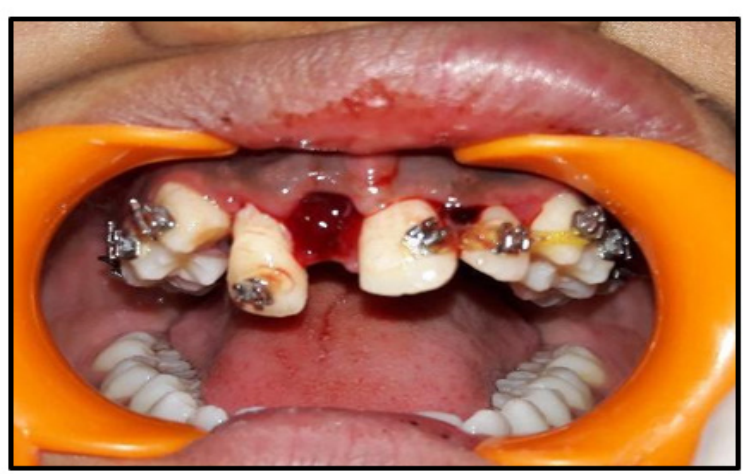

Figure 1: Preoperative.

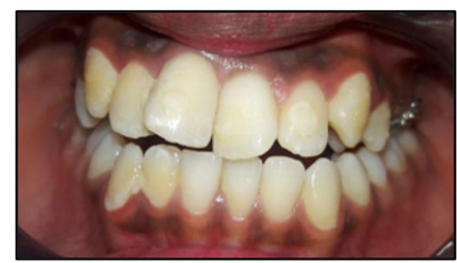

(a)

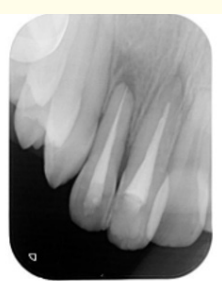

(b)
Figure 2: Post-operative review of the patient after 9 months, clinically (a) and radiographically (b).

instances where replantation is required, the prognosis is better if the tooth can be replanted immediately after the avulsion. If replantation of the tooth cannot be done immediately, it should be stored either in saliva, saline, milk, or Hank's Balanced Salt Solution.

Inflammatory root resorption is seen to be associated with prolonged extraoral dry storage time and the numbers of vital cells are also reduced. In a reported case by Andreasen JO., et al. the physiologic medium used was milk. It was suggested to treat the root surface with substances such as sodium fluoride, tetracycline, stannous fluoride, citric acid, and calcium hydroxide in order to inhibit root resorption [4]. 
Adjunctive therapy in the form of systemic antibiotics given at the time of replantation and prior to endodontic treatment is effective in preventing bacterial invasion of the necrotic pulp and, therefore, subsequent inflammatory resorption. Tetracycline has the added benefit of decreasing root resorption by altering the motility of the osteoclasts and decreasing the effectiveness of collagenase. In addition to the patient's oral hygiene, chlorhexidine rinses should be used for 7 - 10 days [5].

Splinting allows physiologic movement of the tooth (for a minimal time period) during healing and results in a decreased incidence of ankylosis. Flexible splints for 4 weeks have been recommended [6]. Splinting of a replanted tooth to stable adjacent teeth is suggested unanimously in the guidelines and accepted by the clinician. Splinting provides stabilization of replanted teeth through the initial healing period and some movement. Allowing some movement of the replanted tooth is accepted as it has been proved in studies that prolonged rigid splinting of teeth leads to extensive dentoalveolar ankylosis [7].

Orthodontic treatment can cause resorption of the root. Traumatized Teeth that have suffered injuries are more likely to lead to root resorption than non-traumatized teeth [8]. Moving reimplanted teeth orthodontically is possible. According to Malmgren., et al. however reimplantation of permanent teeth after avulsion, a necessary follow-up period of at least one year is essential, since most root resorption occurs during the first year post-trauma [9].

If endodontic therapy is needed following moderate to severe dental trauma, the additional inflammatory stimulation from orthodontic tooth movement may delay the destructive phase acting on the cementum, thereby increasing the likelihood of ankylosis. Therefore, to enable observation of healing and monitoring for ankylosis, orthodontic treatment should be delayed for up to one year [10].

Teeth that are traumatized severely such as those having suffered avulsion or extrusive luxation undergoing orthodontic tooth movement have a poorer prognosis, especially if inflammatory or replacement root resorption occurs. In order to ensure that periodontal healing is complete and to avert extra inflammatory stimuli, which may further damage the protective cementum layer and increase the risk of ankylosis, a rest period of at least one year is therefore recommended [11].

\section{Conclusion}

This case report highlights various factors involving treatment for an avulsed tooth, like duration needed post avulsion usage of doxycycline and treatment for resorption.

Orthodontic treatment for an avulsed tooth is an ongoing debate and challenge to dental practitioners across the world. There are fewer case studies to support the prospect of an equivalent. Further case reports and reviews are required to glean a greater degree of understanding in treating such patients efficiently.

\section{Acknowledgements}

Case reported in M. S. Ramaiah Dental College. Faculty of Dental Sciences. Bangalore.

Guided by Late. Dr. Prahlad Gadicherla, Faculty of Pedodontics, M S Ramaiah Dental College, Bangalore.

\section{Conflict of Interest}

None.

\section{Bibliography}

1. Andreasen JO and Andreasen FM. "Dental traumatology: quo vadis: Opening remarks at the Second International Conference on Oral Trauma, Stockholm, Sweden, September 21, 1989". Dental Traumatology 6.2 (1990): 78-80.

2. Sharma NK and Duggal MS. "Replantation in general dental practice”. British Dental Journal 176.4 (1994): 147.

3. Flores MT., et al. "Guidelines for the management of traumatic dental injuries. II. Avulsion of permanent teeth". Dental Traumatology 23.3 (2007): 130-136.

4. Andreasen JO and Andreasen FM. "Textbook and color atlas of traumatic injuries to the teeth". (4th edn), Blackwell Publishing Company, Denmark, Europe (2007): 1-461.

5. Trope M. "Avulsion of permanent teeth: theory to practice". Dental Traumatology 27.4 (2011): 281-294.

6. Andersson L., et al. "International Association of Dental Traumatology guidelines for the management of traumatic dental injuries: 2. Avulsion of permanent teeth". Dental Traumatology 28.2 (2012): 88-96. 
7. Barrett EJ and Kenny DJ. "Avulsed permanent teeth: a review of the literature and treatment guidelines". Dental Traumatology 13.4 (1997): 153-163.

8. Rocha SRT., et al. "Orthodontic treatment in patients with reimplanted teeth after traumatic avulsion: A case report". Dental Press Journal of Orthodontics 15.4 (2010): 40e1-40e10.

9. Malmgren O., et al. "Abordagem ortodôntica da dentição traumatizada". In: Andreasen JO, Andreasen FM. Texto e atlas colorido de traumatismo dental. $3^{\underline{a}}$ ediion. Porto Alegre: Artmed (2001).

10. Drysdale C., et al. "Orthodontic management of root-filled teeth". British Journal of Orthodontics 23.3 (1996): 255-260.

11. Kindelan SA., et al. "Dental trauma: an overview of its influence on the management of orthodontic treatment. Part 1". Journal of Orthodontics 35.2 (2008): 68-78.

Volume 5 Issue 10 October 2021

(C) All rights are reserved by Zahida Parveen., et al. 